\title{
A 2GHz Delta-Sigma Modulator implemented in InP HBT technology
}

\author{
Guillaume Hincelin \\ CSIRO Telecommunications and Industrial Physics, \\ P O Box 76, Epping, NSW 1710 Australia \\ Tel: (61) 293724378 Fax: (61) 293724488 \\ Guillaume.Hincelin@tip.csiro.au
}

\begin{abstract}
A first-order Delta-Sigma $(\Delta \Sigma)$ modulator has been fabricated using a $70 \mathrm{GHz}\left(f_{T}\right)$ AlInAs/GaInAs-InP HBT technology. At a sampling rate of $2 \mathrm{GHz}$, it converts a 50MHz signal with a maximum signal-to-noise ratio (SNRmax) of $19 \mathrm{~dB}$ and a dynamic range (DR) of $25 \mathrm{~dB}$, equivalent to 3.9DRbits. With a $10 \mathrm{MHz}$ signal, $36.5 \mathrm{~dB} \mathrm{DR}$ (5.8DRbits) and $30.8 \mathrm{~dB}$ SNRmax are achieved. The modulator has been designed in a fully differential architecture, and dissipates $330 \mathrm{~m} W$ from $a-5 V$ power supply. This paper presents the performance of a first-order, continuous-time, low-pass $\Delta \Sigma$ modulator. To date only a few results have been reported for first-order $\Delta \Sigma$ modulator implemented in a III-V technology. A first-order architecture represents a fundamental first step in the design of higher order modulator offering better performance.
\end{abstract}

\section{INTRODUCTION}

High-speed analog-to-digital converters (ADC) are becoming increasingly popular in applications requiring signal processing of wide-band signals. The benefits of using digital signal processing (DSP) over analog processing techniques can be only realised once the wideband signal has been digitised, hence the need for high-speed ADCs. DSP techniques lead to improvements in system cost, system reproducibility and sensitivity to temperature and environmental variations. System flexibility can also benefit as DSP blocks can be reprogrammed to provide new functionality.

The $\Delta \Sigma$ modulation architecture is known for its ability to perform precise analog-to-digital conversion [1, 2]. This technique combines oversampling and noise-shaping to improve ADC resolution: the noise from the quantizer is shaped away from the signal band prior to being filtered in the following stage by a process called decimation.

InP-based HBT technology is used to implement this circuit. The InP process offers many advantages over GaAs process including reduction in the power consumption, improvement in the speed of operation, lower $1 / \mathrm{f}$ noise, lower phase-noise, higher current gain at low current densities and higher thermal conductivity.

The $\Delta \Sigma$ modulation technique when implemented in an InP HBT technology allows the digital conversion of a wideband analog signal to be done at high-speed and with high accuracy. The design of our first-order low-pass continuous-time $\Delta \Sigma$ modulator is described in section 2 and the measured results detailed in the section 3.

\section{CIRCUIT DESIGN}

The aim of this work is to investigate the development of a high frequency $\Delta \Sigma$ modulator to digitise IF frequencies signals in a telecommunications receiver. We have chosen to use a continuous-time implementation [3]. A first-order topology offers a 9dB SNR improvement for each doubling of the sampling frequency. Second and higher order modulators give better performance $[4,5]$ but they are more complex to design and require sharp filtering in the decimation stage. A first-order implementation was undertaken to prove the concept and establish the fundamental design considerations.

The architecture of the system is illustrated in figure 1. A fully differential configuration has been chosen for higher linearity and improved DR. The first-order continuous-time $\Delta \Sigma$ modulator includes a transconductance cell converting a differential voltage to a differential current signal, an integrator to filter the noise away from the amplified signal, a onebit ADC to quantize the signal and a one-bit digital-to-analog converter (DAC) in the feedback loop.

The linearity of the transconductance cell (figure 2) is critical for the overall performance of the system, as it is located at the input to the modulator and outside of the feedback loop. For this reason, two transconductance cells are used together to reduce the distortion to a minimum while maintaining a large flat gain versus frequency performance.

The integrator (figure 3) provides noise shaping as it accumulates the large quantization error from the one-bit ADC. It is formed from a three stage amplifier with two integrating capacitors connected between its input and output. 
The one-bit ADC (figure 4) is a classic comparator using a master-slave architecture and has a significant impact on the final resolution of the $\Delta \Sigma$ modulator. Hysteresis and metastability arising from this component are the limiting factors when the input voltage is decreased or the sampling frequency is increased.

Finally the DAC in the feedback loop is a one-bit current steered differential pair. Two bias current sources are connected to the feedback node to sink the quiescent current of the transconductance cell and DAC.

\section{MEASURED RESULTS}

The fabricated $\Delta \Sigma$ modulator is shown in Figure 5. The circuit includes 88 transistors in a $1.0 \times 1.7 \mathrm{~mm}^{2}$ die size. It operates from a $-5 \mathrm{~V}$ power supply and dissipates $330 \mathrm{~mW}$.

The measurements were completed on wafer using a high frequency Cascade probe card. Observations of the pseudorandom digital output were captured on a $20 \mathrm{GSp} / \mathrm{s}$ Agilent digital oscilloscope. Figure 6 shows an eye diagram of a $50 \mathrm{MHz}$ input signal sampled at $2 \mathrm{GHz}$. Two Anritsu synthesizers are used for the clock and the $\Delta \Sigma$ modulator inputs. $2 \mathrm{GHz}$ was found to be the maximum rate at which the $\Delta \Sigma$ modulator could be clocked. Above this frequency, the rise and fall time necessary for the $\Delta \Sigma$ modulator to switch correctly from one level to the other start to exceed the sampling period. This limitation is due to the metastability of the one-bit ADC and can be improved by adding a third stage of latch buffering after the master-slave ADC.

Figure 7 shows two output spectrums sampled with a $500 \mathrm{MHz}$ and a $2 \mathrm{GHz}$ clock frequency. The performance was measured with a HP spectrum analyser. The noise at the lower frequency is clearly shaped away to higher frequency and the SNR of the $50 \mathrm{MHz}$ input signal is improved with increasing clock frequency. We also notice harmonic signals at multiples of the signal frequency. These tones are visible at large input amplitudes, (in this case $0 \mathrm{dBm}$ ). These spurious are likely to be limit cycle tones directly related to the quantization error. First order modulators are known to be more susceptible to limit cycle tones than higher-order topologies. These tones arise because the quantisation error is correlated with the input signal. It is important to take this into account, as linear models of $\Delta \Sigma$ modulators do not predict limit cycle tones. They assume the quantization error to be uncorrelated white noise. A solution is to add dither to the input signal to force it to be "busy" but with an associated reduction in SNR [6].

To evaluate the performances of the $\Delta \Sigma$ modulator, the SNR (including distortion tones) has been measured with a range of input power values at different clock frequencies. The results are plotted in figure 8 . With a $50 \mathrm{MHz}$ signal, the SNRmax are $7.6 \mathrm{~dB}, 13.4 \mathrm{~dB}$ and $19 \mathrm{~dB}$ for $500 \mathrm{MHz}, 1 \mathrm{GHz}$ and $2 \mathrm{GHz}$ sampling frequencies respectively. Measurements were also made for a $10 \mathrm{MHz}$ signal. For the same sampling frequencies, SNRmax of 21.9dB, 26.4dB, 30.8dB are obtained respectively. The ideal improvement of the SNR at a specific input magnitude for each doubling clock frequency is about $9 \mathrm{~dB}$ for a first-order $\Delta \Sigma$ modulator. In practice, we obtain up to $8 \mathrm{~dB}$ improvement.

The DR corresponds to the input amplitude range for which the SNR is positive in the linear area. For the $50 \mathrm{MHz}$ bandwidth signal, its value is about 9,19 and $25 \mathrm{~dB}$ for $500 \mathrm{MHz}, 1 \mathrm{GHz}$ and $2 \mathrm{GHz}$ clock frequencies respectively. This corresponds to 1.2, 2.9 and 3.9DRbits. Better dynamic ranges are obtained for the narrower $10 \mathrm{MHz}$ signal bandwidth as the oversampling ratio is increased. For the same sampling frequencies, the DR is approximately $28 \mathrm{~dB}, 32.5 \mathrm{~dB}$, and $36.5 \mathrm{~dB}$ respectively, representing $4.3,5.1$ and 5.8DRbits.

\section{CONCLUSION}

A first-order, continuous-time $\Delta \Sigma$ modulator has been successfully implemented in an AlInAs/GaInAs-InP HBT technology. At a sampling rate of $2 \mathrm{GHz}$, it converts a $50 \mathrm{MHz}$ signal bandwidth with a SNRmax of $19 \mathrm{~dB}$ and a DR of $25 \mathrm{~dB}$, equivalent to 3.9DRbits. With a $10 \mathrm{MHz}$ signal, 36.5dB DR (5.8DRbits) and 30.8dB SNRmax are achieved at the same sampling frequency. The circuit presented in this paper indicates the potential for $\Delta \Sigma$ modulators to provide highspeed digitisation of IF frequencies in a telecommunications receiver. This design represents the first stage of our development of high-speed high-order $\Delta \Sigma$ modulators.

\section{ACKNOWLEDGEMENTS}

The author thanks P. Roberts, S. Giugni, J. Bunton, P. Vun, L. Stokes, J. Archer and M. Kelly for their valuable help. Thanks are also due to TRW in Los Angeles who fabricated the MMIC.

\section{REFERENCES}

[1] J. C. Candy and G. C. Temes. Oversampling methods for $A / D$ and $D / A$ conversion. Oversampling DeltaSigma Data Converters: Theory, Design, and Simulation, IEEE Press, New York, 1992. 
[2] S. R. Norsworthy et al. Delta-sigma data converters: Theory, Design, and Simulation. IEEE Press, New York, 1997.

[3] J.A. Cherry. Theory, practice, and fundamental performance limits of high-speed data conversion using continuous-time delta-sigma modulators. Ph.D. thesis, Carleton University, 1998.

[4] J. F. Jensen et al. A 3.2-GHz second-order delta-sigma modulator implemented in InP HBT technology. IEEE J. Solid-State Circ., pages 1119-1127, October 1995.

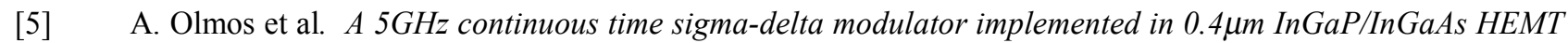
technology. Proc. Int. Symp. Circ. Syst., volume 1, pages 575-578, 1998.

[6] M. F. Wagdy. Effect of various dither forms on quantization errors of ideal A/D converters. IEEE Trans Instru. Measu., volume 38, No 4, pages 850-855, 1989.

\section{FIGURES}

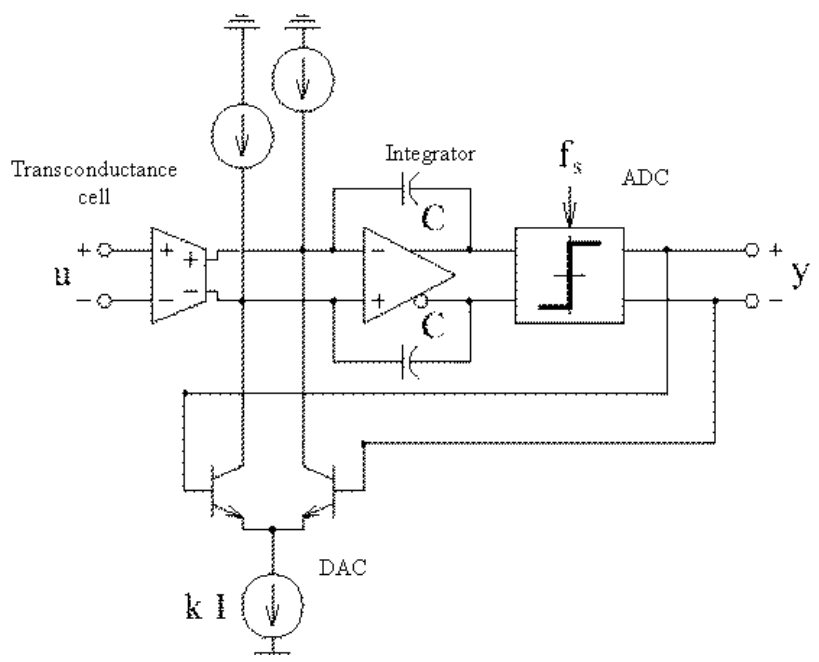

Figure 1. Simplified diagram of the first-order $\Delta \Sigma$ modulator.

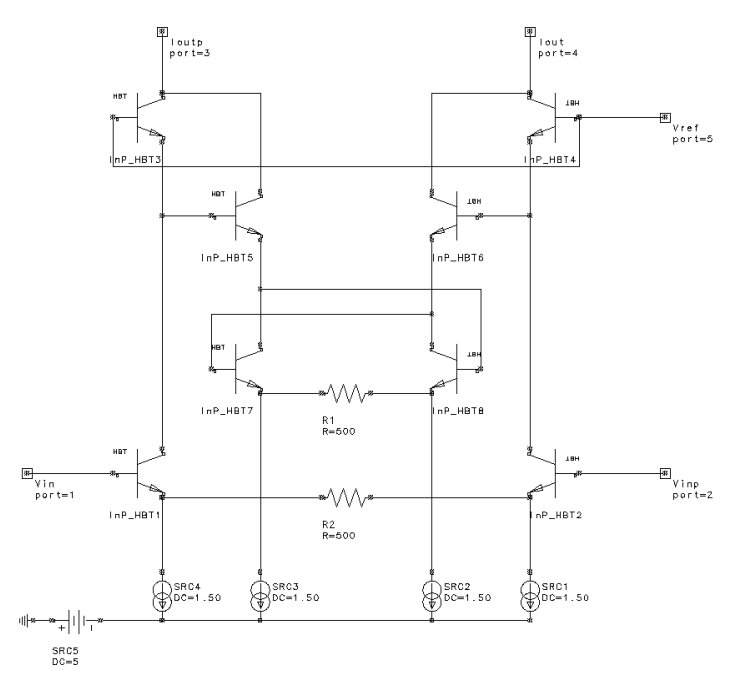

Figure 2. Schematic diagram of the transconductance cell.

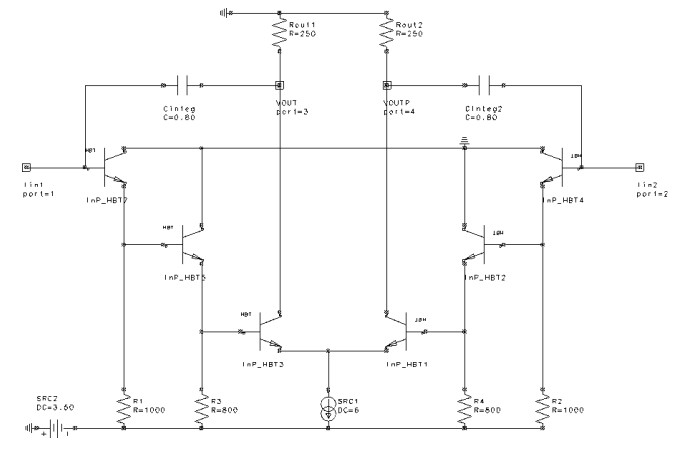

Figure 3. Schematic diagram of the integrator.

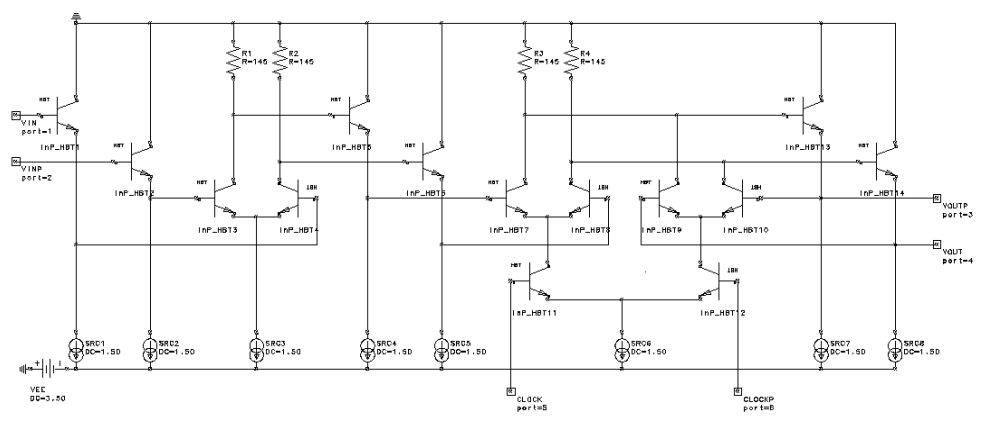

Figure 4. Schematic diagram of the ADC (with master stage only). 


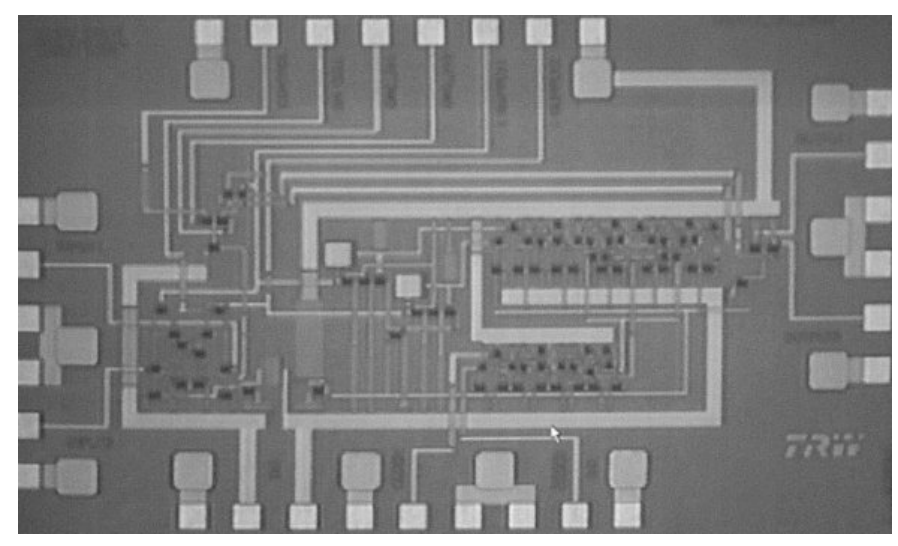

Figure 5. Die photograph of the first-order $\Delta \Sigma$ modulator.

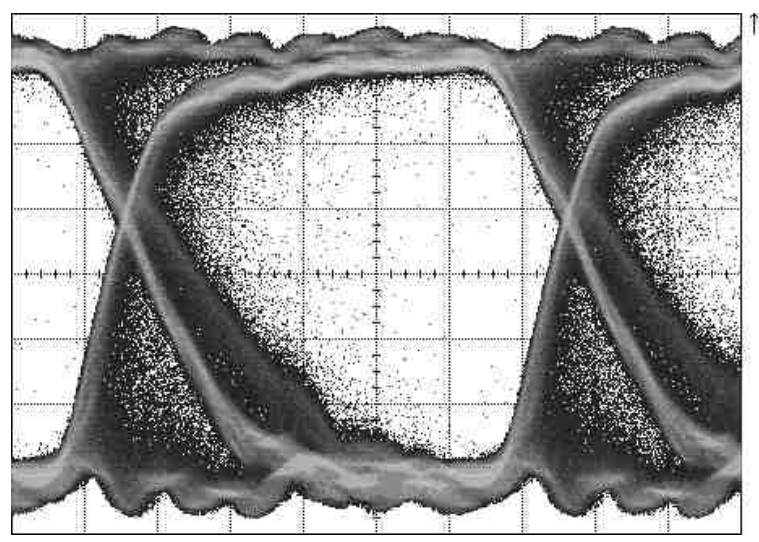

Figure 6. Eye diagram of a $50 \mathrm{MHz}$ signal sampled at $2 \mathrm{GHz}$.

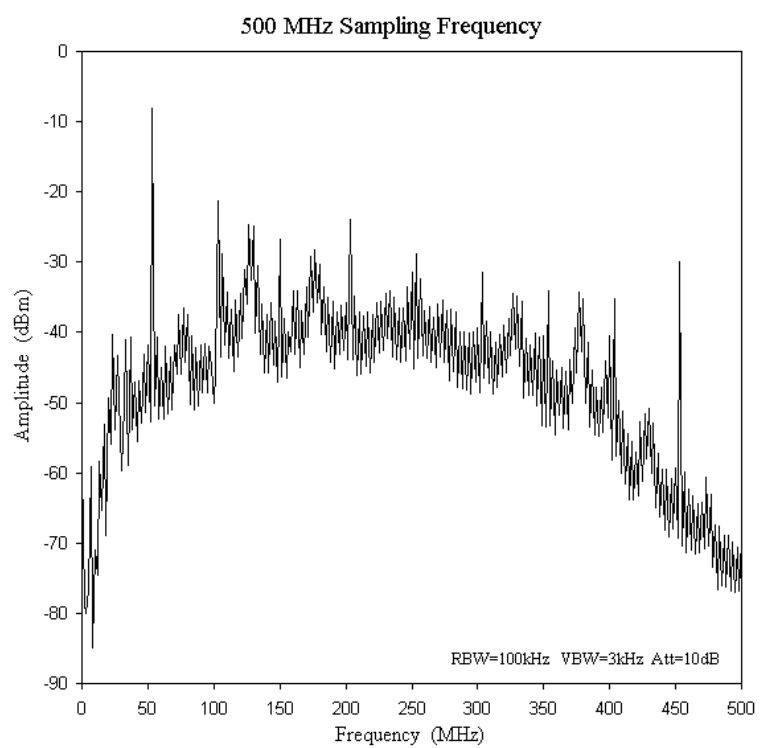

(a)

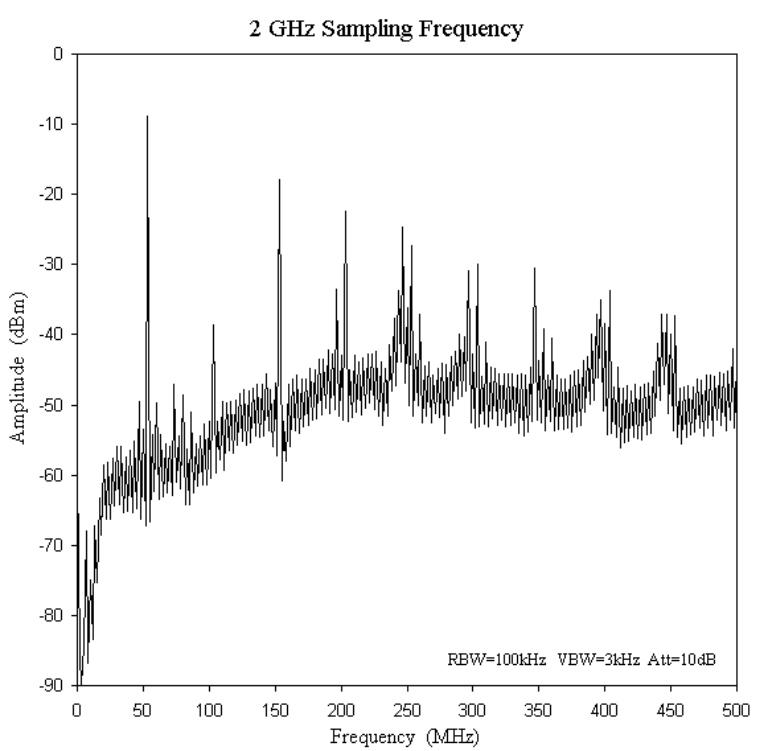

(b)

Figure 7. Frequency spectra measured from a $50 \mathrm{MHz}$ and $0 \mathrm{dBm}$ input signal at clock frequencies of (a) $500 \mathrm{MHz}$ and (b) $2 \mathrm{GHz}$.

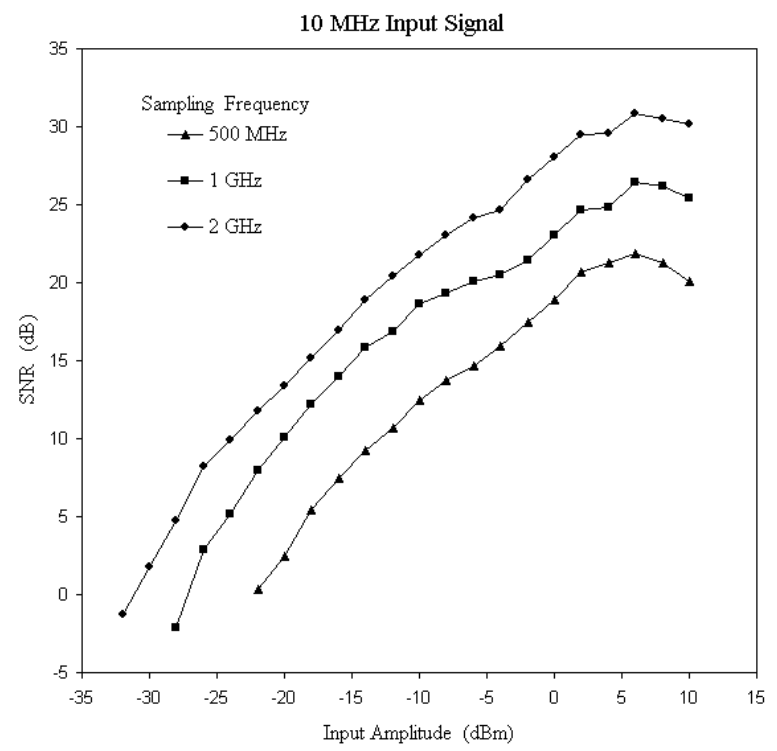

(a)

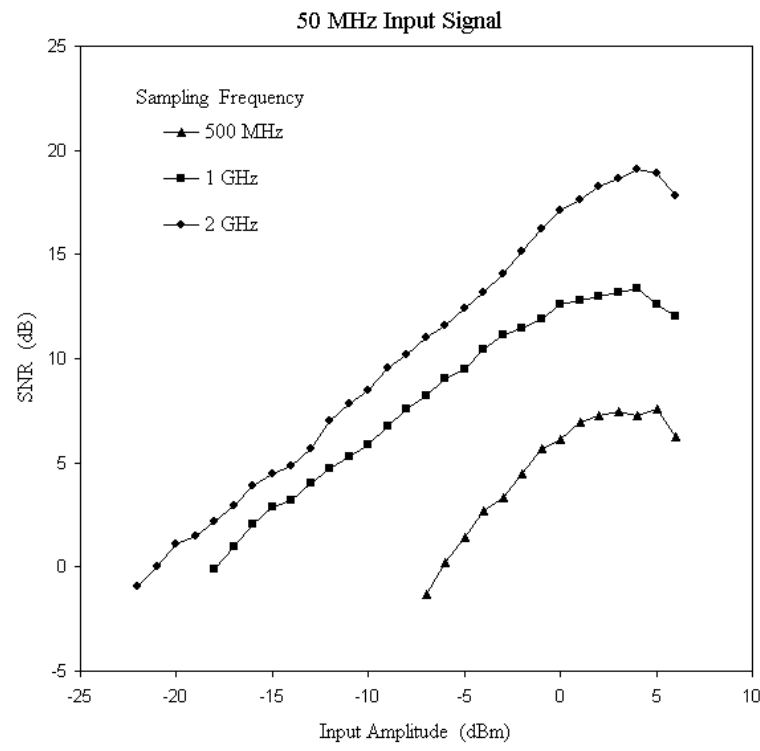

(b)

Figure 8. SNR obtained from a (a) $10 \mathrm{MHz}$ and (b) $50 \mathrm{MHz}$ signal with three clock frequencies, $500 \mathrm{MHz} 1 \mathrm{GHz}$ and $2 \mathrm{GHz}$. 